TITLE:

\title{
Correlations of intra- and intermolecular dynamics and structure in liquid para-hydrogen
}

$\operatorname{AUTHOR}(S)$ :

Kim, Hyeon-Deuk; Ando, Koji

\section{CITATION:}

Kim, Hyeon-Deuk ...[et al]. Correlations of intra- and intermolecular dynamics and structure in liquid para-hydrogen. Physical Review B 2014, 90(16): 165132.

ISSUE DATE:

2014-10

URL:

http://hdl.handle.net/2433/191209

RIGHT:

(C)2014 American Physical Society 
PHYSICAL REVIEW B 90, 165132 (2014)

\title{
Correlations of intra- and intermolecular dynamics and structure in liquid para-hydrogen
}

\author{
Kim Hyeon-Deuk ${ }^{1,2, *}$ and Koji Ando ${ }^{1}$ \\ ${ }^{1}$ Department of Chemistry, Kyoto University, Kyoto 606-8502, Japan \\ ${ }^{2}$ Japan Science and Technology Agency, PRESTO, 4-1-8 Honcho, Kawaguchi, Saitama 332-0012, Japan \\ (Received 17 August 2014; revised manuscript received 6 October 2014; published 27 October 2014)
}

\begin{abstract}
Although hydrogen is the simplest of all molecular species, its nuclear quantum effects dominate the structure and thermodynamical properties of the condensed phases. With a recently developed nonempirical quantum molecular dynamics simulation method, we present intuitive understandings of real-time dynamics of each para-hydrogen $\left(p-\mathrm{H}_{2}\right)$ molecule in the liquid phase including its $\mathrm{H}-\mathrm{H}$ bond vibrations, molecular orientations, and librational motions. The short- and long-living memories of angular dynamics we found suggest that $p-\mathrm{H}_{2}$ should be described as a librating nonspherical diatomic molecule and can be characterized by two typical dynamics: kinetic motions inside a solvation shell and diffusive dynamics out of the shell. The real-time trajectory reveals that structural rearrangements of a $p-\mathrm{H}_{2}$ molecule such as breakout from a solvation shell significantly correlate with its intramolecular structure and fluctuations. The $\mathrm{H}-\mathrm{H}$ bond power spectra completely fitted by two functions also indicate that liquid $p-\mathrm{H}_{2}$ has two typical structures. The condensed-phase effects on shifts and broadening of $\mathrm{H}-\mathrm{H}$ bond stretching frequencies, intrinsic librational dynamics, and molecular orientational distributions are computationally demonstrated and physically rationalized.
\end{abstract}

DOI: 10.1103/PhysRevB.90.165132

PACS number(s): 67.63.Cd, 67.10.Hk, 83.10.Rs

Understanding microscopic dynamics of condensed-phase systems exhibiting significant quantum effects is still one of the open problems in condensed matter materials. Hydrogen is the simplest of all molecular species although nuclear quantum effects (NQEs) dominate the properties of the condensed phases. Liquids composed of such quantum particles are called quantum liquids, and they show phenomena which have not been observed for ordinary classical liquids.

The NQEs of liquid para-hydrogen $\left(p-\mathrm{H}_{2}\right)$ appear as a broader distribution of radial distribution functions (RDFs), the large self-diffusion coefficients even at low temperatures, and the anomalous temperature dependence of thermal conductivity and shear viscosity [1-8]. A number of quantum mechanical and dynamical approaches have been examined to elucidate the anomalous tendency observed in this quantum liquid. A complete treatment of the quantum dynamics for large systems using basis set methods requires storage of multidimensional wave functions and computational effort that grows exponentially with the number of degrees of freedom. Instead, semiquantum methods suitable for numerical simulations of real $p-\mathrm{H}_{2}$ ensembles have been proposed based on the imaginary-time path-integral theory $[1-3,5,6,9-13]$. These provide either the static properties or the equilibrium time correlation functions (TCFs) accounting for particularly important NQEs such as zero-point energy effect to evaluate the transport properties over a wide temperature range $[1-6,12,13]$. All these previous methods unanimously adopted the Silvera-Goldman(SG) model potential [14] that assumes that the $p-\mathrm{H}_{2}$ molecules are a spherically symmetric particle in the rotational ground state. Because the critical point temperature $33.1 \mathrm{~K}$ is substantially lower than the first rotational excitation temperature $87.6 \mathrm{~K}$, the vast majority of $p-\mathrm{H}_{2}$ molecules in their ground rotational state were assumed to be modeled by the isotropic SG potential. However, the spherical symmetry of each hydrogen molecule in the

*Corresponding author: kim@kuchem.kyoto-u.ac.jp liquid state is by no means established. No orientational ordering in a low-pressure solid hydrogen only guarantees the uniform angular distribution in the whole system [15]. In fact, symmetry breaking accompanying the phase transition is related to the molecular orientational ordering [16-18]. It is also suggested that the angular degrees of freedom and $\mathrm{H}-\mathrm{H}$ vibrational frequency can be used to judge crystallization and monitor a temperature of clusters [19].

We recently proposed a simulation method of nuclear and electron wave packet molecular dynamics (NEWPMD) based on nonempirical $a b$ initio intra- and intermolecular interactions of nonspherical hydrogen molecules that can be applied to condensed-phase $p-\mathrm{H}_{2}$ with feasible computational cost [20,21]. Even long-range dispersion interactions depending on intermolecular angles, which density functional theory without Hartree-Fock-type exchange terms cannot describe, were successfully reproduced [21]. Our method nonperturbatively accounts for the important quantum features of a $p-\mathrm{H}_{2}$ system, while offering an intuitive understanding of microscopic real-time dynamics of each $p-\mathrm{H}_{2}$ molecule including its angular and intramolecular degrees of freedom. This is a report on microscopic structures and dynamics of $p-\mathrm{H}_{2}$ molecules in a condensed-phase liquid $p-\mathrm{H}_{2}$.

The NEWPMD approach describes nuclei by floating and breathing Gaussian WPs via the time-dependent Hartree approach, and EWPs by the perfect-pairing valence bond theory that appropriately treats the Pauli exclusion energy and intermolecular dispersion energy [20]. While the Gaussian widths of EWPs were fixed in our previous NEWPMD simulation [20], the current version allows them to freely change depending on the $\mathrm{H}-\mathrm{H}$ bond length $r_{\mathrm{HH}}(t): 0.398+0.360 r_{\mathrm{HH}}(t) \AA$ for the large EWPs, and $0.176+0.244 r_{\mathrm{HH}}(t) \AA$ for the small EWPs. These linear dependencies of $\rho_{k}(t)$ on $r_{\mathrm{HH}}(t)$ are deduced from the NEWP calculation on a $\mathrm{H}_{2}$ molecule [21]. We adopt these thawed EWPs to calculate all of the intramolecular potentials. We thus call this method flexible-NEWPMD(F-NEWPMD) to distinguish it from the previous version developed in Ref. [20]. 

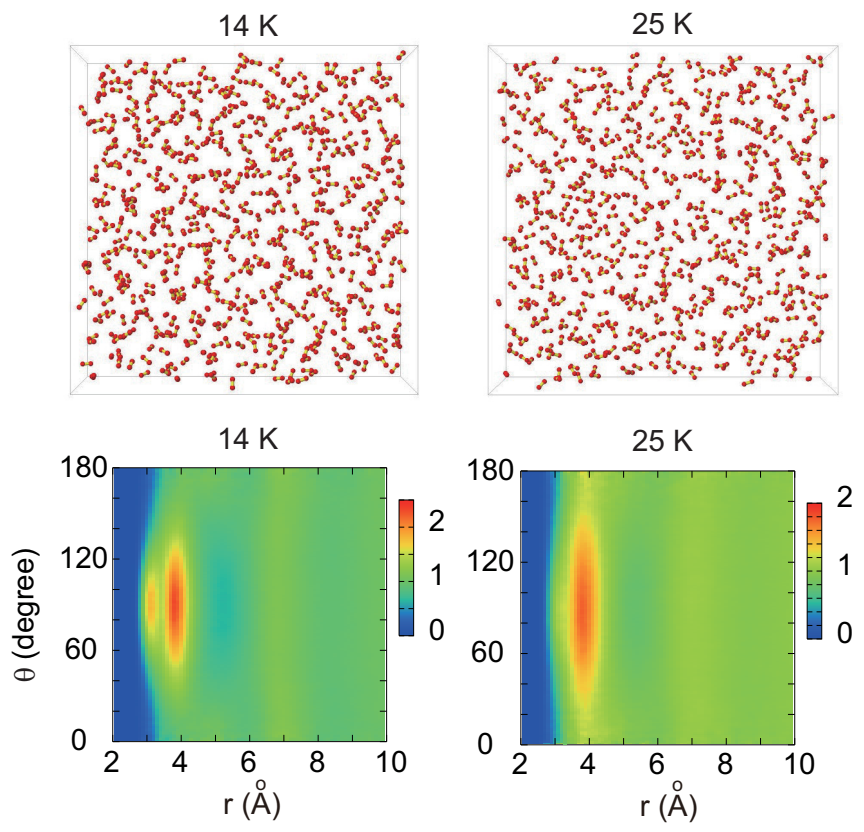

FIG. 1. (Color online) (upper) Top views of the 3D cubic simulation cells containing $576 p-\mathrm{H}_{2}$ molecules at 14 and $25 \mathrm{~K}$. The corresponding real-time movies are given as Supplementary Movies. The real-time dynamics include the $\mathrm{H}-\mathrm{H}$ bond vibrations, molecular orientations, and librational dynamics of each $p-\mathrm{H}_{2}$ molecule. (lower) 2D RDFs as a function of distance between centers of two hydrogen NWPs, $r$, and an intermolecular bond angle, $\theta$. The relative angle $\theta$ symmetrically distributes near 90 degrees. The shoulder appearing in the first RDF peak of $14 \mathrm{~K}$ reflects the diatomic structure of a $\mathrm{H}_{2}$ molecule in the T-shape solvation shell, while the shoulder almost disappears at $25 \mathrm{~K}$ due to the thermal modulations.

The computational costs for the current F-NEWPMD are reasonable; propagating 576 molecules for 1 ps requires 26 minutes on a single core of a Xeon CPU with $2.60 \mathrm{GHz}$.

The top views of the three-dimensional (3D) cubic simulation boxes containing $576 p-\mathrm{H}_{2}$ molecules at 14 and $25 \mathrm{~K}$ are shown in the upper panels of Fig. 1, respectively. The liquid $p-\mathrm{H}_{2}$ is set to have the molar volume at the saturated vapor pressure, $27.0 \times 10^{-6} \mathrm{~m}^{3} / \mathrm{mol}$ at $14 \mathrm{~K}$ and $32.0 \times 10^{-6} \mathrm{~m}^{3} / \mathrm{mol}$ at $25 \mathrm{~K}$ [2]. As shown in Fig. S1, the F-NEWPMD successfully reproduces the basic properties of the liquid $p-\mathrm{H}_{2}$ such as the RDFs and self-diffusion coefficients [22]. The corresponding real-time movies are given as Supplementary Movies [22]. The real-time dynamics of condensed $p-\mathrm{H}_{2}$ molecules including the $\mathrm{H}-\mathrm{H}$ bond vibrations, molecular orientations, and librational dynamics are visualized in the movies. To characterize the liquid $p-\mathrm{H}_{2}$ structure in terms of the relative molecular orientations, the two-dimensional (2D) RDF [23]

$$
g(r, \theta)=\frac{\langle n(r, \theta)\rangle}{2 \pi n_{0} r^{2} d r \sin \theta d \theta}
$$

was calculated. Here, $r$ is the distance between centers of two hydrogen NWPs, and $\theta$ is a relative angle formed by a pair of molar vectors of two $\mathrm{H}_{2}$ molecules. $\langle n(r, \theta)\rangle$ represents the average number of NWP centers in $[r, r+d r]$ and $[\theta, \theta+d \theta]$, while $n_{0}$ is the net number density. The resulting
2D RDFs at 14 and $25 \mathrm{~K}$ are given in the lower panels of Fig. 1. The shoulder appearing in the left part of the first $\mathrm{RDF}$ peak at $14 \mathrm{~K}$ reflects the diatomic structure of a $\mathrm{H}_{2}$ molecule in the first solvation shell; the most stable solvation structure is the T-shape configuration, and the nearest atoms lead to the shoulder around $3.15 \AA$. In fact, this shoulder exhibits the sharper peak along the angular coordinate $\theta$ than the main peak near $3.85 \AA$; the structural fluctuations are smaller in the nearest hydrogen atoms forming the stiff T-shape configurations. This shoulder reflecting the diatomic structure will be found if the more detailed structure factor with a larger wave vector is observed by the neutron scattering experiments. The second peak around $7.05 \AA$ has the peak close to 0 and 180 degrees at $14 \mathrm{~K}$ although the peak is too small to see in Fig. 1. This is because the second nearest $\mathrm{H}_{2}$ molecule tends to be parallel to the central $\mathrm{H}_{2}$ molecule. We note that, however, hydrogen molecules are free rotors in the whole system as shown by the uniform distribution of $\theta$ in Fig. S6(a) [15]. In the region of larger radial distance, the distribution along the angular coordinate $\theta$ becomes more uniform, and the correlation between the radial and angular coordinates decays. The shoulder in the first RDF peak almost disappears at $25 \mathrm{~K}$ because of the larger configurational fluctuations. The higher temperature also induces the broader distribution along the angular coordinate $\theta$. This less-structured T-shape configuration implies softer librational motions of $\mathrm{H}_{2}$ molecules at $25 \mathrm{~K}$ than at $14 \mathrm{~K}$, which will be demonstrated in Fig. 2(c).

The F-NEWPMD includes the intramolecular interactions of a nonspherical $\mathrm{H}_{2}$ molecule and can describe detailed microscopic structures of a $\mathrm{H}_{2}$ molecule even in a condensed phase. Figure 2(a) shows the distributions of the H-H bond length $r_{\mathrm{HH}}$. The distributions are fitted by the single Gaussian functions whose parameter values are listed in Table S1 [22]. The average $\mathrm{H}-\mathrm{H}$ bond length, $0.7659 \AA$ at $14 \mathrm{~K}$ and $0.7655 \AA$ at $25 \mathrm{~K}$, are reasonably compared to the experimental result, $0.755 \AA[21,24]$. The average $\mathrm{H}-\mathrm{H}$ bond length in the liquid phase is longer than the bond length of the isolated free molecule, $0.7643 \AA$, which is nontrivial but agrees with the experimental finding in the low-pressure solid [24]. Because the most stable solvation structure is the T-shape configuration, the $\mathrm{H}-\mathrm{H}$ bond of a molecule is effectively elongated by the attraction from the electron distribution in the bonding region of the adjacent $\mathrm{H}_{2}$ molecules as the system becomes more condensed. In fact, the $\mathrm{H}-\mathrm{H}$ bond is more stretched at $14 \mathrm{~K}$ than at $25 \mathrm{~K}$. On the contrary, the width of the $r_{\mathrm{HH}}$ distribution at $25 \mathrm{~K}, 4.60 \times 10^{-3} \AA$, is twice as large as that at $14 \mathrm{~K}, 2.30 \times 10^{-3} \AA$, reflecting the larger thermal fluctuations at $25 \mathrm{~K}$ than at $14 \mathrm{~K}$. The average NWP widths are correlated with the $\mathrm{H}-\mathrm{H}$ bond length as in Fig. S2; as the $\mathrm{H}-\mathrm{H}$ bond stretches, the NWP becomes delocalized, and vice versa [22]. The corresponding TCFs and power spectra of the $\mathrm{H}-\mathrm{H}$ bond length $r_{\mathrm{HH}}(t)$ are shown in Figs. S3 and 2 (b), respectively $[22,25]$. The power spectrum of $r_{\mathrm{HH}}(t)$ at $14 \mathrm{~K}$ exhibits the shoulder and is almost fitted by the equal combination of the two Gaussian functions whose parameter values are given in Table S1. The H-H bond frequency of the main peak, $4604.7 \mathrm{~cm}^{-1}$, overestimates the experimental data at $14.2 \mathrm{~K}, 4151.5 \mathrm{~cm}^{-1}$ [19] by $10.9 \%$ that would be almost irrelevant to the discussions in this paper. The Gaussian function with the lower average frequency, $4602.0 \mathrm{~cm}^{-1}$, and 


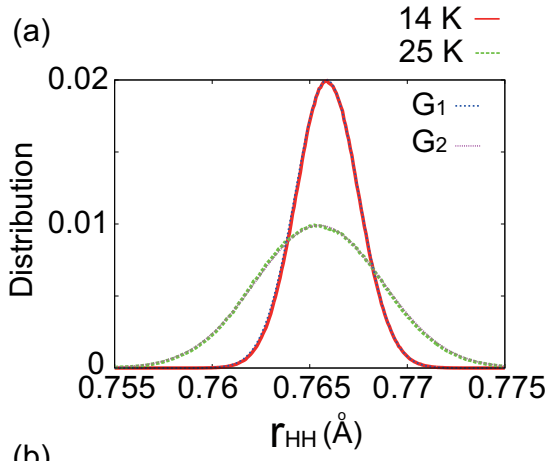

(b)

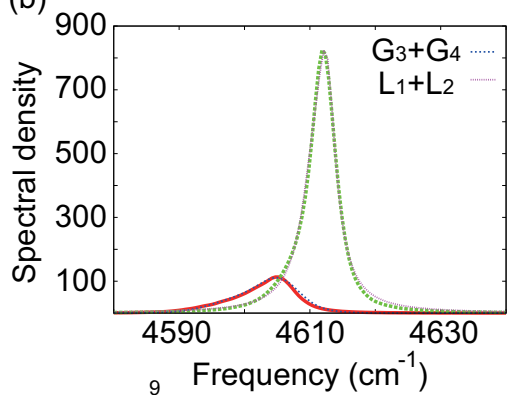

(c)

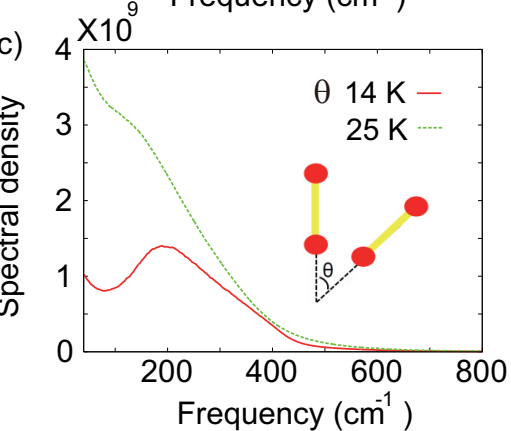

FIG. 2. (Color online) (a) Distributions of the H-H bond length $r_{\mathrm{HH}}$ in the $p-\mathrm{H}_{2}$ liquid. (b) Power spectra of the $\mathrm{H}-\mathrm{H}$ vibrations. The power spectra involve the two $\mathrm{H}-\mathrm{H}$ stretchings of the well-structured and less-structured $\mathrm{H}_{2}$ molecule. The shrinked $\mathrm{H}-\mathrm{H}$ bond at $25 \mathrm{~K}$ leads to the blueshift. The motional narrowing due to the fast vibrational dephasings is observed at $25 \mathrm{~K}$. (c) Power spectra of the intermolecular angle $\theta$. The rigid solvation structure at $14 \mathrm{~K}$ leads to the higher librational frequency, $200 \mathrm{~cm}^{-1}$, while the partially broken configuration at $25 \mathrm{~K}$ results in the red-shifted librational frequency, $150 \mathrm{~cm}^{-1}$.

the broader width, $7.9 \mathrm{~cm}^{-1}$, corresponds to the $\mathrm{H}-\mathrm{H}$ vibration of the well-structured $\mathrm{H}_{2}$ molecule; the $\mathrm{H}-\mathrm{H}$ bond is stretched in the rigid structure as discussed above, and, due to the resulting weakened bond potential, the vibrational frequency is red-shifted and broadened. The other Gaussian function with the higher average frequency, $4605.5 \mathrm{~cm}^{-1}$, and the narrower width, $3.9 \mathrm{~cm}^{-1}$, corresponds to the $\mathrm{H}-\mathrm{H}$ vibration of the less-structured $\mathrm{H}_{2}$ molecule. The fact that the total power spectrum can be fitted by only the two kinds of the Gaussian functions indicate that the liquid $p-\mathrm{H}_{2}$ at $14 \mathrm{~K}$ has two typical structures. On the other hand, the power spectrum of $r_{\mathrm{HH}}(t)$ at $25 \mathrm{~K}$ is fitted by the equal combination of the two Lorentzian functions. This fact indicates that the liquid $p-\mathrm{H}_{2}$ at $25 \mathrm{~K}$ is also composed of two typical structures just as at $14 \mathrm{~K}$ but involves faster vibrational dephasings to induce the motional narrowing. In fact, the larger thermal fluctuations at the higher temperature result in about eight times more intense spectrum in $25 \mathrm{~K}$ than in $14 \mathrm{~K}$. Only $11 \mathrm{~K}$ temperature increase causes this qualitative difference in the $\mathrm{H}-\mathrm{H}$ stretching modes. The blue-shifted $\mathrm{H}-\mathrm{H}$ bond frequency of the main peak for $25 \mathrm{~K}$, $4611.9 \mathrm{~cm}^{-1}$, compared to the $14 \mathrm{~K}$ case is correlated with the shorter $\mathrm{H}-\mathrm{H}$ bond discussed for Fig. 2(a). In the isolated free molecule case, the $\mathrm{H}-\mathrm{H}$ bond is most shrinked and the power spectrum of $r_{\mathrm{HH}}(t)$ has no shoulder but exhibits the single sharp peak around $4629.2 \mathrm{~cm}^{-1}$ (Fig. S4 [22]). The calculated redshift of $24.5 \mathrm{~cm}^{-1}$ from the peak of the isolated case to the main peak of the $14 \mathrm{~K}$ case is comparable to the experimental redshift from the isolated case to the $14.2 \mathrm{~K}$ case, $9.6 \mathrm{~cm}^{-1}[19,26]$. Correlations between the H-H stretching mode and the NWP beating motion are also observed in Fig. S5 [22].

Our real-time simulation provides information on intra- and intermolecular angles of nonspherical hydrogen molecules in the liquid-phase $p-\mathrm{H}_{2}$. We focus on the intramolecular angle $\phi$ as well as the intermolecular angle $\theta$. The logarithmic TCFs of $\phi$ and $\theta$ exhibit the short- and long-time scales (Figs. S6(b) and S6(c) [22]). The ratio of the deduced shorter timescales, $120 \mathrm{fs}$ over $250 \mathrm{fs}$, is almost proportional to the ratio of the temperatures, $14 \mathrm{~K}$ over $25 \mathrm{~K}$, suggesting that these timescales are related to kinetic dynamics of a $\mathrm{H}_{2}$ molecule inside a small first solvation shell. The ratio of the deduced longer timescales, 225 fs over $1590 \mathrm{fs}$, is more sensitive to the temperature and rather close to the ratio of the diffusion coefficients for $14 \mathrm{~K}$ and $25 \mathrm{~K}, 0.287 \AA^{2} /$ ps over $1.496 \AA^{2} /$ ps [20]. This coincidence indicates that these longer timescales reflect diffusive dynamics of a $\mathrm{H}_{2}$ molecule out of solvation shells. The finite-timescale memories of $\theta$ dynamics demonstrate that angular dynamics of $\mathrm{H}_{2}$ molecules cannot be neglected even at the current temperatures which are lower than the rotational temperature. Figure 2(c) shows the power spectra of $\theta(t)$ whose peaks appear near 200 and $150 \mathrm{~cm}^{-1}$ at 14 and $25 \mathrm{~K}$, respectively. We attribute these peaks in the low-frequency region to librations of $\mathrm{H}_{2}$ molecules rather than to rotational motions. Supplementary Movies confirm this conclusion as one can see mostly librational motions during the $14 \mathrm{ps}$ movies but only a few rotational motions. The temperature dependence of the peak frequency and intensity also supports the current libration picture; the rigid T-shape structure at $14 \mathrm{~K}$ provides a sharp librational potential leading to the higher peak frequency of librations. At $25 \mathrm{~K}$, the T-shape configuration is partially broken and the librational potential becomes softened, resulting in the red-shifted librational motions. The spectral intensity for $25 \mathrm{~K}$ is generally larger than the intensity for $14 \mathrm{~K}$ due to the stronger thermal fluctuations of librations. The rotational region of Raman spectra for liquid $\mathrm{p}-\mathrm{H}_{2}$ is almost independent of the temperature [19], and, further, the current temperatures are too low to cause larger-amplitude and unidirectional rotational motions. We extracted and elucidated the librational motions of $\mathrm{H}_{2}$ molecules in the condensed liquid phase. The above insights are further supported by the power spectra of the intramolecular angle $\phi(t)$ [Fig. S6(d)].

To demonstrate the significant correlations among the interand intramolecular dynamics, Fig. 3 displays the real-time trajectory of a representative $\mathrm{H}_{2}$ molecule at $25 \mathrm{~K}$ focusing on the square displacement, the intramolecular angles $\phi$ and 

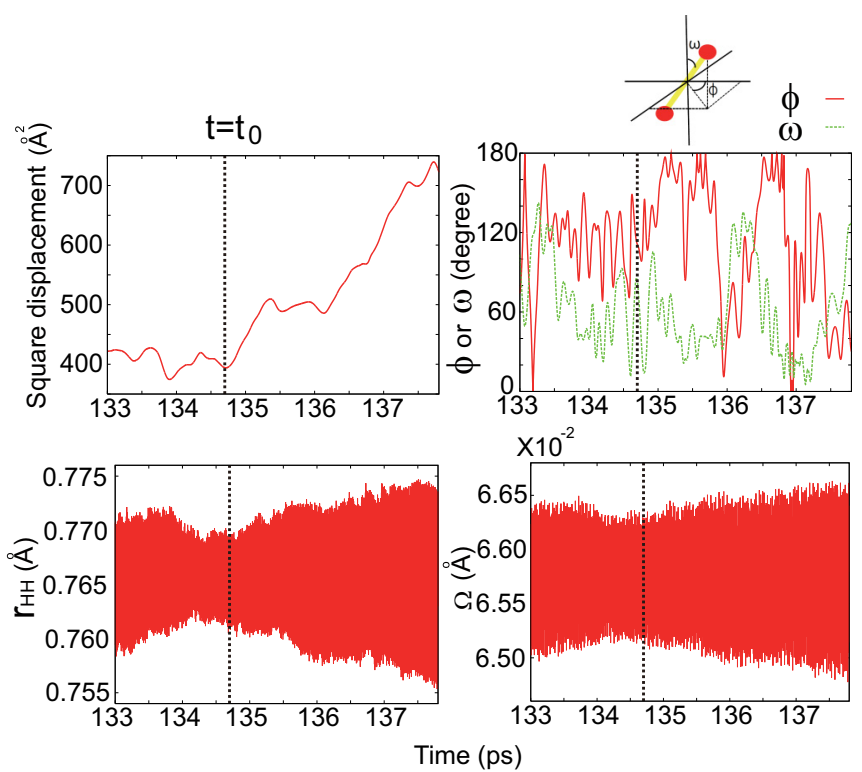

FIG. 3. (Color online) Real-time dynamics of a representative $\mathrm{H}_{2}$ molecule in the $p-\mathrm{H}_{2}$ liquid at $25 \mathrm{~K}$. The vertical line in each panel divides the time window at $t=t_{0}$. The square displacement (upper left), the intramolecular angles $\phi$ and $\omega$ (upper right), the $\mathrm{H}-\mathrm{H}$ bond length (lower left), and the NWP width (lower right) are all significantly correlated.

$\omega$, the $\mathrm{H}-\mathrm{H}$ bond length, and the NWP width. All of the panels in Fig. 3 show the same time window in which the square displacement of the representative $\mathrm{H}_{2}$ molecule exhibits the significant change, about $7 \AA$ displacement. The significant displacement means structural rearrangements such as breakout from a solvation shell; for $t<t_{0}$, the representative molecule stays in a stable structure and its square displacement is stably fluctuating, while, for $t>t_{0}$, the molecule breaks a solvation shell and drastically moves for a long range. The large displacement in $t>t_{0}$ induces the significant fluctuations of the intramolecular angles $\phi$ and $\omega$, while in $t<t_{0}$ they stably oscillate by librations inside a solvation shell; the translational motion of the $\mathrm{H}_{2}$ molecule is directly coupled to its angular dynamics. The large displacement is also accompanied by shrinking of the average $\mathrm{H}-\mathrm{H}$ bond length and enhancement of the $\mathrm{H}-\mathrm{H}$ bond fluctuations. During the large displacement in $t>t_{0}$, the molecule is almost free from the attractive interactions in the solvation shell, leading to the decrease in the average $\mathrm{H}-\mathrm{H}$ bond length. The increase in the $\mathrm{H}-\mathrm{H}$ bond fluctuations provides further evidence for this freedom that the representative molecule undergoes. On the other hand, in $t<t_{0}$, the $\mathrm{H}_{2}$ molecule is rigidly trapped in a solvation shell and its $\mathrm{H}-\mathrm{H}$ bond is elongated due to the attractive force from the surrounding shell molecules as discussed in Fig. 2(a), while the $\mathrm{H}-\mathrm{H}$ bond fluctuations are suppressed due to the stiff shell structure and the restricted free molar space formed by the solvating molecules. The NWP width exhibits the similar dynamics to the $\mathrm{H}-\mathrm{H}$ bond stretching (Fig. S4). Although the above correlations are not complete, reflecting complicated fluctuations with coupling to other variables, they are seen not only in the shown time window but also many times in the entire duration of the simulation. The $14 \mathrm{~K}$ case is presented in Fig. S7 [22].

This paper provided the intuitive understandings of realtime dynamics of each nonspherical $p-\mathrm{H}_{2}$ molecule in the liquid phase including its $\mathrm{H}-\mathrm{H}$ bond vibrations, angular motions, and NWP beating motions. The insights obtained here indicate that condensed-phase hydrogens constitute a class of molecular quantum liquids that should be characterized by anisotropic molecules with angular and intramolecular degrees of freedom. Our work has initiated a quantitative trace of $p-\mathrm{H}_{2}$ single molecule dynamics even in a condensed phase and has wide scope for extensions in various phases and thermodynamic states including symmetry breaking accompanying a phase transition that is related to orientational ordering. Directly obtained information on angular degrees of freedom and $\mathrm{H}-\mathrm{H}$ vibrational frequency for each $p-\mathrm{H}_{2}$ will play an essential role in judging and monitoring crystallization as well as a temperature of condensed-phase hydrogens.

K.H.D. acknowledges financial support from JST (PRESTO) and Grant-in-Aids for Scientific Research from Japan Society for the Promotion of Science (KAKENHI), Grant No. 24750016. K.A. acknowledges support from KAKENHI No. 22550012 and No. 26620007.
[1] D. Scharf, G. J. Martyna, and M. L. Klein, Fiz. Nizk. Temp. 19, 516 (1993) [Low Temp. Phys. 19, 364 (1993)].

[2] Y. Yonetani and K. Kinugawa, J. Chem. Phys. 120, 10624 (2004).

[3] Y. Yonetani and K. Kinugawa, J. Chem. Phys. 119, 9651 (2003).

[4] F. J. Bermejo, K. Kinugawa, C. Cabrillo, S. M. Bennington, B. Fak, M. T. Fernandez-Diaz, P. Verkerk, J. Dawidowski, and R. Fernandez-Perea, Phys. Rev. Lett. 84, 5359 (2000).

[5] F. J. Bermejo, B. Fak, S. M. Bennington, K. Kinugawa, J. Dawidowski, M. T. Fernandez-Diaz, C. Cabrillo, and R. Fernandez-Perea, Phys. Rev. B 66, 212202 (2002).

[6] M. Zoppi, M. Neumann, and M. Celli, Phys. Rev. B 65, 092204 (2002).

[7] F. J. Bermejo, B. Fak, S. M. Bennington, R. Fernandez-Perea, C. Cabrillo, J. Dawidowski, M. T. Fernandez-Diaz, and P. Verkerk, Phys. Rev. B 60, 15154 (1999).
[8] D. Colognesi, M. Celli, M. Neumann, and M. Zoppi, Phys. Rev. E 70, 061202 (2004).

[9] A. Nakayama and N. Makri, J. Chem. Phys. 119, 8592 (2003).

[10] J. A. Poulsen, G. Nyman, and P. J. Rossky, J. Phys. Chem. B 108, 19799 (2004).

[11] J. Liu and W. H. Miller, J. Chem. Phys. 128, 144511 (2008).

[12] T. F. Miller and D. E. Manolopoulos, J. Chem. Phys. 122, 184503 (2005).

[13] I. Georgescu, J. Deckman, L. J. Fredrickson, and V. A. Mandelshtam, J. Chem. Phys. 134, 174109 (2011).

[14] I. F. Silvera and V. V. Goldman, J. Chem. Phys. 69, 4209 (1978).

[15] G. Geneste, M. Torrent, F. Bottin, and P. Loubeyre, Phys. Rev. Lett. 109, 155303 (2012).

[16] H. Kitamura, S. Tsuneyuki, T. Ogitsu, and T. Miyake, Nature (London) 404, 259 (2000).

[17] H. Mao and R. J. Hemley, Rev. Mod. Phys. 66, 671 (1994). 
[18] I. I. Mazin, R. J. Hemley, A. F. Goncharov, M. Hanfland, and H.-k. Mao, Phys. Rev. Lett. 78, 1066 (1997).

[19] R. Sliter and A. F. Vilesov, J. Chem. Phys. 131, 074502 (2009).

[20] K. Hyeon-Deuk and K. Ando, J. Chem. Phys. 140, 171101 (2014).

[21] K. Hyeon-Deuk and K. Ando, Chem. Phys. Lett. 532, 124 (2012).

[22] See Supplemental Material at http://link.aps.org/supplemental/ 10.1103/PhysRevB.90.165132 for the real-time movies of condensed-phase $p-\mathrm{H}_{2}$, and additional figures for intra- and intermolecular structures and dynamics of condensed-phase $p-\mathrm{H}_{2}$.

[23] K. Kasahara and H. Sato, J. Chem. Phys. 140, 244110 (2014).

[24] P. Loubeyre, M. Jean-Louis, and I. F. Silvera, Phys. Rev. B 43, 10191 (1991).

[25] S. Saito and I. Ohmine, J. Chem. Phys. 102, 3566 (1995).

[26] W. Kolos and L. Wolniewicz, J. Chem. Phys. 49, 404 (1968). 